\title{
Editores:
}

Fernanda Garcia Sampaio

Consuelo Marques da Silva

Renato Hiroshi Torigoi

Luciene Mignani

Ana Paula Contador Packer

Celso Vainer Manzatto

Juliana Lopes da Silva

\section{Estratégias de}

Monitoramento Ambiental

\section{da Aquicultura}

Portfólio de Resultados do Monitoramento Ambiental da Aquicultura em Águas da União

Secretaria de Agricultura e Abastecimento Agência Paulista de Tecnologia dos Agronegócios Instituto de Pesca

Rede Nacional de Pesquisa e Monitoramento Ambiental da Aquicultura em Águas da União 


\section{EXPEDIENTE}

João Doria

Governador do Estado de São Paulo

Gustavo Diniz Junqueira

Secretário de Agricultura e Abastecimento

Antonio Batista Filho

Coordernador da Apta

Vander Bruno dos Santos

Diretor Técnico de Departamento do Instituto de Pesca

Rede Nacional de Pesquisa e Monitoramento Ambiental da Aquicultura em Águas da União

Direção Nacional

Juliana Lopes da Silva

Coordenação Técnico Científica

Fernanda Garcia Sampaio

\section{Editores}

Fernanda Garcia Sampaio

Consuelo Marques da Silva

Renato Hiroshi Torigoi

Luciene Mignani

Ana Paula Contador Packer

Celso Vainer Manzatto

Juliana Lopes da Silva

Fotos da Capa:

Glauber Pereira de Santos Carvalho

Gustavo Henrique Gonzaga da Silva

Fotos contra capa interna:

Fernanda Garcia Sampaio

Gustavo Henrique Gonzaga da Silva

Felipe Schwahofer Landuci

Projeto e editoração eletrônica:

Paulo Eduardo Marchezini

Dados Internacionais de Catalogação na Publicação (CIP)

Elaborada pelo Núcleo de Informação e Documentação. Instituto de Pesca, São Paulo S862e Sampaio, Fernanda Garcia et al.

Estratégias de monitoramento ambiental da aquicultura: portifólio de resultados do monitoramento ambiental da aquicultura Em água da União. - São Paulo, 2019.

iv, 95.; il..gráf.

ISBN 978-65-80646-00-5

1. Nanoecologia. 2. Nanomateriais. 3. Agrotóxicos. 4. Consumo de oxigênio. 5. Excreção de amônia. I. Sampaio, Fernanda Garcia.

CDD 574.3

Permitida a cópia parcial, desde que citada a fonte 


\section{Avaliação Ambiental do Cultivo da Macroalga Marinha Hypnea Pseudomusciformis no Litoral do Ceará}

Wagner Cotroni Valenti', Stefany Almeida Pereira' e Janaina Mitsue Kimpara².

${ }^{1}$ Centro de Agricultura da Universidade Estadual Paulista; ${ }^{2}$ Empresa Brasileira de Pesquisa Agropecuária Embrapa Meio Norte.

A concentração de nutrientes ( $C, N$ e $P$ ) e a taxa de sedimentação de materiais em suspensão na do entorno foram monitorados para a avaliação da sustentabilidade da atividade produtiva por meio da aplicação de indicadores.

\section{ÁREA DE ESTUDO}

Praia de Flecheiras, município de Trairi, Cearó Foi implantado um cultivo experimental da macroalga nativa (Hypnea pseudomusciformis). sistema consistia em 2 long-lines de 15 metros de cada $20 \mathrm{~cm}$ de corda na superficienarradas cada 20 cal de corda, na supericie da água. a 45 dias.

\section{METODOLOGIA}

Amostras de água e de material sedimentado na area do cultivo e a $100 \mathrm{~m}$ de distância (controle) foram coletadas a cada 15 dias. O materia sedimentado foi amostrado utilizando um coletor de tripton amarrado a $1 \mathrm{~m}$ de profundidade $\mathrm{do}$ long-line do culfivo de macroalgas, por 24 horas. $O$ cáculo da taxa de sedimentaçáo foi feito com base na area do coletor e o tempo de coleta. material sedimentado foi analisado quanto a fósforo total pelo método de colorimetria do metavanadato (Michelsen, 1957), e quanto a carbono orgânico total e nitrogennio total, em analisado Vario TOC Select (Elementar $\circledast$ ). A agua foi analisada quanto a concentraçáo de N-nitrito, N-nitrato, reagentes e espectrofotômetro Hach, modelo 1900 (Hach, Colorado, EUA)

Para determinação do fósforo total na água, as amostras foram submetidas a uma digestão previa de acordo com o metodo de digestão persulfato te, P-ortofosato foi medido 5 ) peterormenreto estanhoso (APHA 2005 - método 4500-P.D). com auxílio de espectrofotômetro digital (Shimadzu UV-1800 (). As variáveis avaliadas foram comSigmaSTAT 4.0, considerando um valor de $\mathrm{P}<0,05$.

RESULTADOS PRINCIPAIS

A partir das informações obtidas, podemos in ferir que:

- Não houve diferença estatisticamente significativa entre o cultivo e o controle para as variáveis analisadas.

- O material sedimentado sob o cultivo corespondeu a material inorgânico, especialmente areia, carregada pela corrente marítima.

- Não há uso de insumos poluentes no cultivo de macroalgas.

RESULTADO

- O cultivo de macroalgas pode ser considerado uma afividade que não altera significativamente a qualidade da água nessa escala de produção.

\section{SOLUÇÕES}

- A atividade de produção de macroalgas marinhas poderia ser classificada como sendo de baixo impacto, por resultar em mudanças não significativas na água de cultivo e na sedimentação de escala de produção, a origem autóctone da espécie e o não requerimento de insumos externos que podem causar poluição aquática, o licenciamento ambiental para esta atividade poderia ser dispensado ou simplificado, mediante comprovação de planejamento da produção que não comprometa os estoques naturais.

\section{DESAFIOS}

- No Brasil, estudos futuros devem ser realizados para avaliar externalidades associadas ao materiais em suspensão. Observando-se a devida cultivo comercial de macroalgas, tanto positiva quanto negativas, como, por exemplo, o potencia de redução de acidificação costeira e a biorre-

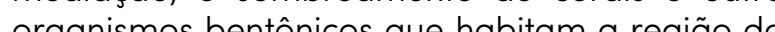
cultivos e a reduçã de queques naturais.

- A Resolução CONAMA N 357/2005 considera águas salinas aptas para cultivo de organismos aquáticos as de Classe 1. Os valores das variáveis analisadas estão acima dos limites para classifica- ção das águas Classe 1 descritos na Resolução. No entanto, para fins de licenciamento, e importante que hajútrico em que o empreendimento aquicola será instalado, para que o mesmo não modifique c ambiente a ponto de afetar sua resiliencia. Se for considerado o parâmetro da Resolução, não seria possivel qualquer atividade quícola marinha naquela região. Portanto, há o desafio de se adequar a regulamentação para licenciamento ambienta de atividades aquicolas marinhas no Brasil.

Tabela 1. Médias e desvios padrão das variáveis monitoradas

\begin{tabular}{l|c}
\multicolumn{1}{c}{ Variáveis } & Controle \\
\hline Carbono orgânico total $\left(\mathrm{mg} \mathrm{L}^{-1}\right)$ & $10,19 \pm 0,00$ \\
\hline Sólidos em suspensão $\left(\mathrm{g} \mathrm{L} \mathrm{L}^{-1}\right)$ & $0,04 \pm 0,04$ \\
\hline Fósforo total $\left(\mathrm{mg} \mathrm{L}^{-1}\right)$ & $4,30 \pm 0,50$ \\
\hline Nitrato $\left(\mathrm{mg} \mathrm{L}^{-1}\right)$ & $1,30 \pm 0,56$ \\
\hline Nitrito $\left(\mathrm{mg} \mathrm{L}^{-1}\right)$ & $0,00 \pm 0,00$ \\
\hline Nitrogênio total $\left(\mathrm{mg} \mathrm{L}^{-1}\right)$ & $0,17 \pm 0,00$ \\
\hline Ortofosfato solúvel $\left(\mathrm{mg} \mathrm{L}^{-1}\right)$ & $0,05 \pm 0,09$
\end{tabular}

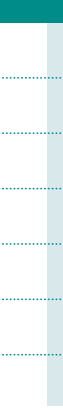

\begin{tabular}{cc}
\hline $10,22 \pm 0,83$ & 3,00 \\
$0,06 \pm 0,03$ & - \\
\hline $4,93 \pm 1,99$ & 0,06 \\
$1,18 \pm 0,62$ & 0,40 \\
\hline $0,00 \pm 0,00$ & 0,07 \\
$0,07 \pm 0,03$ & - \\
$0,05 \pm 0,07$ & - \\
\hline
\end{tabular}

*Conforme Resolução CONAMA № 357/2005, de 17 de março de 2005.

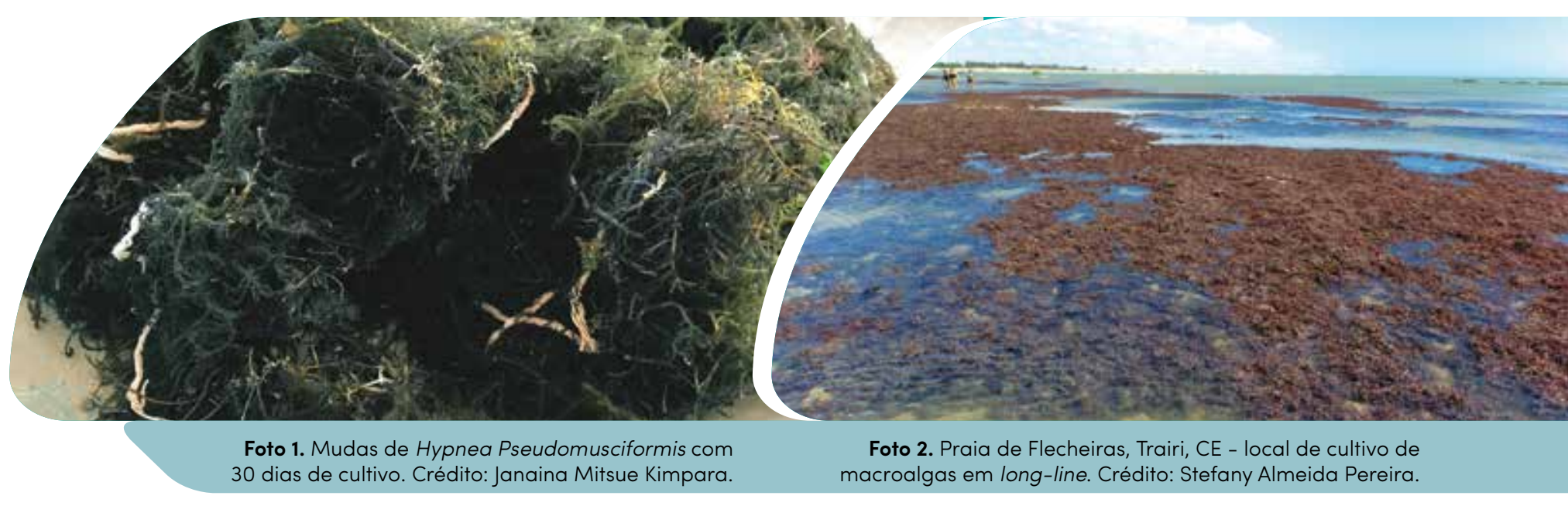

Dr. Wagner Cotroni Valenti - Universidade Estadual Paulista - e-mail

Dra. Janaina Mitsue Kimpara - Empresa Brasileira de Pesquisa Agropecuária - Embrapa Meio Norte - e-mail: janaina.kimpara@embrapa.br
REFERÊNCIAS:

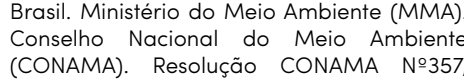
18/03/2005. Dispoe sobre a classificaçaco dos o seu vnauadramento, bem como estabelece

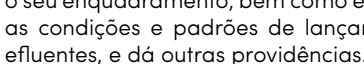

